\title{
Trigonometric and Gaussian Quadrature
}

\author{
By C. J. Knight and A. C. R. Newbery
}

\begin{abstract}
Some relationships are established between trigonometric quadrature and various classical quadrature formulas. In particular Gauss-Legendre quadrature is shown to be a limiting case of trigonometric quadrature.
\end{abstract}

In an earlier paper [1] it was noted that there exist trigonometric and exponential analogs of Gaussian quadrature formulas. We now extend those results to show several interesting features. We find that Legendre and trapezoidal rule quadrature result from the trigonometric formula as a certain parameter takes special values. We also study in some detail a case in which the roots of an orthogonal polynomial tend to coalesce. The development is based on transforming the integrand into Gaussian form. The problem then reduces to finding a polynomial belonging to an orthogonal set. An algorithm is proposed for constructing the polynomial's coefficients numerically.

We will restrict our consideration to a quadrature formula of the form

$$
\int_{-1}^{1} f(x) d x=\sum_{i=1}^{n} \beta_{i} f\left(x_{i}\right)+T,
$$

where $T$ is the truncation error. The formula is to be exact (i.e., $T=0$ ) when $f(x)$ belongs to a function space with basis

$$
\{1, \cos \omega x, \cdots, \cos (n-1) \omega x\} .
$$

Note that we have assumed $f(x)$ is an even function in writing (2). We can append a set of linearly independent odd functions to obtain a basis set for a more general integrand, say $g(x)$. The choice of the odd basis set is arbitrary since the integration is over symmetric limits. We will discuss two choices. The parameter $\omega$, which may be complex, is to be chosen for convenience in each problem. We will only consider $\omega$ real with $\omega \in[0, \pi]$ or imaginary with $\omega=i \sigma$ and $\sigma \in[0, \infty)$.

First suppose that

$$
g(x)=\sum_{0}^{n-1} a_{r} \cos r \omega x+\sum_{1}^{n-1} b_{r} \sin r \omega x .
$$

The reduction to Gaussian form in this case is essentially given in [1], but we now incorporate the parameter $\omega$ into the approximant rather than parametrize the range of integration. Writing $u=\tan (\omega x / 2) / t$ with $t=\tan (\omega / 2)$ and noting that

Received July 17, 1969, revised November 10, 1969.

AMS Subject Classifications. Primary 6555; Secondary 4205.

Key Words and Phrases. Trigonometric quadrature, Gaussian quadrature, orthogonal polynomials, coalescing roots, orthogonal polynomials, construction of orthogonal polynomials. 
$\sin \omega x=2 t u /\left(1+t^{2} u^{2}\right)$ and $\cos \omega x=\left(1-t^{2} u^{2}\right) /\left(1+t^{2} u^{2}\right)$, we find $g(x)=Q(u ; \omega) /\left(1+t^{2} u^{2}\right)^{n-1}$ with $Q$ a polynomial in $u$ of order $2 n-2$. Consequently,

$$
\int_{-1}^{1} g(x) d x=\frac{2 t}{\omega} \int_{-1}^{1} \frac{Q(u ; \omega) d u}{\left(1+t^{2} u^{2}\right)^{n}} .
$$

It follows from [2, pp. 331-334] that the integral on the right in (3) can be evaluated by an $n$-point quadrature formula with nodes at the zeros of $q_{n}(y ; \omega, n)$, where $\left\{q_{r}\right\}$ is the set of monic polynomials orthogonal with respect to the inner product

$$
\langle F, G\rangle=\int_{-1}^{1} \frac{F(u) \cdot G(u) d u}{\left(1+t^{2} u^{2}\right)^{n}} .
$$

Another choice of the set of basis functions leads to a more convenient reduction to Gaussian form. To motivate our choice note that the basis (2) is not convenient for small values of $\omega$ because it tends to be linearly dependent. An alternate choice of the even basis functions is provided by the

LemMa. For any $\phi$ and integer $m$

$$
\left[\cos \left(\frac{\phi}{2}\right)\right]^{2 m} \equiv 2^{-2 m+1}\left\{\cos m \phi+\left(\begin{array}{c}
2 m \\
1
\end{array}\right) \cos (m-1) \phi+\cdots+\frac{1}{2}\left(\begin{array}{c}
2 m \\
m
\end{array}\right)\right\},
$$

where $\left(\begin{array}{l}k \\ l\end{array}\right)$ are the binomial coefficients. $(C f .[3, p .461)$.)

If we set $\phi=\pi-\omega x$ and note that $\cos (\pi / 2-\psi)=\sin \psi$ and $\cos (k \pi-\psi)=$ $(-1)^{k} \cos \psi$ we find (2) is taken into a new basis set by the linear transformation

$$
\left[\frac{\sin (\omega x / 2)}{s}\right]^{2 m}=2(2 s)^{-2 m}\left\{(-1)^{m} \cos m \omega x+\cdots+\frac{1}{2}\left(\begin{array}{c}
2 m \\
m
\end{array}\right)\right\},
$$

with $s=\sin (\omega / 2)$ and $m=0,1, \cdots, n-1$. An obvious choice of a basis for the odd components of $g$ is now the odd powers of $\sin (\omega x / 2) / s$ of order one through $2 n-3$. Then as $|\omega| \rightarrow 0$ we find the complete basis set

$$
\left\{1, \cdots,[\sin (\omega x / 2) / s]^{2 n-2}\right\} \rightarrow\left\{1, x, \cdots, x^{2 n-2}\right\}+O\left(|\omega|^{2}\right),
$$

and it obviously remains linearly independent.

Gautschi [4] has previously used this method to replace the basis (2) by a basis differing from (5) only by a multiplicative constant. We would like to thank the referee for pointing this out.

Suppose that $g(x)=\sum_{0}^{2 n-2} c_{r}[\sin (\omega x / 2)]^{r}$. Introducing $y=\sin (\omega x / 2) / s$ reduces $g$ to a polynomial in $y$ of order $2 n-2$, say $P(y ; \omega)$, and our integral becomes

$$
\int_{-1}^{1} g(x) d x=\frac{2 s}{\omega} \int_{-1}^{1} \frac{P(y ; \omega) d y}{\left(1-s^{2} y^{2}\right)^{1 / 2}}
$$

The integral on the right in (6) is again of Gaussian form. Let $\left\{p_{r}\right\}$ be the set of monic polynomials orthogonal with respect to the inner product

$$
(F, G)=\int_{-1}^{1} \frac{F(y) \cdot G(y) d y}{\left(1-s^{2} y^{2}\right)^{1 / 2}} .
$$

Then from [2, pp. 331-334] we know it can be evaluated by an $n$-point quadrature formula with nodes at the zeros of $p_{n}(y ; \omega)$. 
Note that although the discussion has proceeded as if $\omega$ were real, the inner products (4) and (7) remain real for $\omega$ imaginary. It follows that $q_{n}$ and $p_{n}$ will have $n$ real, simple zeros in $(-1,1)$ for $\omega$ real or imaginary.

The polynomial sets $\left\{q_{r}\right\}$ and $\left\{p_{r}\right\}$ are fundamentally different. They are connected only by the fact that the zeros of $q_{n}(u ; \omega, n)$ and the zeros of $p_{n}(y ; \omega)$ must lead to the same nodes $x_{i}$ in (1). This establishes the following relationship between corresponding zeros of the two polynomials:

$$
u_{i}=y_{i} \cos (\omega / 2) /\left[1-s^{2} y_{i}^{2}\right]^{1 / 2} .
$$

Note that $u_{i}$ vanishes like $\cos (\omega / 2)$ as $\omega \rightarrow \pm \pi$, and $u_{j} \rightarrow y_{i}$ as $\omega \rightarrow 0$. We will prefer to work with the set $\left\{p_{r}\right\}$.

Before considering the actual construction of $p_{n}$, let us study the quadrature formula qualitatively by allowing $\omega$ to approach special values. The form of the inner product (7) makes rather obvious the first theorem.

THEOREM 1. Let $\left\{P_{r}\right\}$ and $\left\{T_{r}\right\}$ be the set of monic Legendre and Chebyshev polynomials, respectively. Then for any integer $r$ and with $y=\sin (\omega x / 2) / \sin (\omega / 2)$, as $\omega \rightarrow 0$ along the real or imaginary axis

$$
p_{r}(y ; \omega)=P_{r}(x)+O\left(|\omega|^{2}\right),
$$

and as $\omega \rightarrow \pi$ along the real axis

$$
p_{r}(y ; \omega)=T_{r}(y)+O(\pi-\omega)^{2} .
$$

Proof. The theorem follows by applying the Gram-Schmidt procedure, noting that the inner product (7)

$$
(F, G)=\int_{-1}^{1} F(y) \cdot G(y) d y+O\left(\left|\omega^{2}\right|\right) \quad \text { as }|\omega| \rightarrow 0
$$

and

$$
(F, G)=\int_{-1}^{1} \frac{F(y) \cdot G(y) d y}{\left(1-y^{2}\right)^{1 / 2}}+O(\pi-\omega)^{2} \quad \text { as } \omega \rightarrow \pi,
$$

to show that $p_{r} \rightarrow P_{r}(y)$ or $T_{r}(y)$ in the respective cases. We then need only note (5) to show that $P_{r}(y)=P_{r}(x)+O\left(|\omega|^{2}\right)$ as $|\omega| \rightarrow 0$.

It follows from this theorem that Legendre quadrature is a special case of the quadrature formula (1). We also know from [5] that (1) should reduce to the trapezoidal rule as $\omega \rightarrow \pi$. To show this is true let us note that the roots of the $n$th order Chebyshev polynomial are $\cos [(2 m-1) \pi / 2 n], m=1,2, \cdots, n$. Thus, for $\omega=\pi$ the nodes in (1) become

$$
x_{m}=\frac{2}{\pi} \sin ^{-1}\left\{\cos \left[\frac{(2 m-1) \pi}{2 n}\right]\right\}=\frac{n+1-2 m}{n} .
$$

These are precisely the nodes of the trapezoidal rule.

It is interesting to note that the nodes of the trapezoidal rule are more centrally located than the Legendre nodes. This realization together with extensive numerical experiments leads us to make the following conjecture.

Conjecture 1. Each nonzero node $x_{i}$ of the quadrature formula (1) moves mono- 
tonically inward from the Legendre node to the trapezoidal rule node as $\omega$ increases from zero to $\pi$. The movement is not strictly monotone at $\omega=0$.

No counterexample to this conjecture has been found in a set of tests where $n$ ranged from 2 to 10 and $\omega$ from $10^{-4}$ to $\pi-10^{-4}$. The exceptional nature of the case $\omega=0$ is shown in

THEOREM 2. Each node $x_{i}$ in (1), considered as a function of $\omega$, is stationary at $\omega=0$.

Proof. We have, from Theorem $1, p_{n}=P_{n}+\omega^{2} p+O\left(|\omega|^{4}\right)$, where $p(x)$ is a polynomial. Let $z_{j}$ be a zero of $P_{n}(x)$ and $z_{i}+\delta x_{i}$ be a zero of $p_{n}$. Then,

$$
p_{n}\left(z_{i}+\delta x_{i}\right)=P_{n}\left(z_{j}+\delta x_{i}\right)+\omega^{2} p\left(z_{j}+\delta x_{j}\right)+O(|\omega|)^{4}=0 .
$$

Since $P_{n}\left(z_{i}\right)=0$ and $P_{n}^{\prime}\left(z_{i}\right) \neq 0$ we have $\delta x_{i}=-\omega^{2} p\left(z_{i}\right) / P_{n}^{\prime}\left(z_{i}\right)+O\left(|\omega|^{4}\right)$, and hence $d x_{i} / d \omega=0$ at $\omega=0$.

Since the nodes are stationary with respect to $\omega$ at $\omega=0$, we can assume their variation with $\omega$ is slow for small $|\omega|$. This means there is little purpose in using (1) for small $|\omega|$; we would get almost the same result by Legendre quadrature. In the case of trigonometric quadrature this conclusion is intuitively reasonable. If the assumed period of the integrand is very long relative to the range of integration, it is easy to believe the (aperiodic) polynomial approximant would do as well as (1).

We will next consider the behavior of our quadrature formula with $\omega=i \sigma$ as $\sigma \rightarrow \infty$. The treatment will be based on the system of equations given by the method of undetermined coefficients. Before deriving these equations it is convenient to note that (1) must be a symmetric quadrature formula because the integral is over symmetric limits. Thus, defining $N$ as the greatest integer less than or equal to $n / 2$, (1) can be rewritten as

$$
\int_{-1}^{1} f(x) d x=\sum_{i=1}^{N} \beta_{i}\left[f\left(x_{i}\right)+f\left(-x_{i}\right)\right] \text { for } n=2 N
$$

and

$$
\int_{-1}^{1} f(x) d x=\beta_{0} f(0)+\sum_{i=1}^{N} \beta_{i}\left[f\left(x_{i}\right)+f\left(-x_{i}\right)\right] \text { for } n=2 N+1 .
$$

We will choose the $x,>0$. Recall that these formulas are to be exact when $f \in\{1$, $\cosh \sigma x, \cdots, \cosh (n-1) \sigma x\}$. Substituting each basis function into (9) then gives the systems of equations

$$
\frac{\sinh m \sigma}{m \sigma}=\sum_{i=1}^{N} \beta_{i} \cosh m \sigma x_{i}, \quad m=0,1, \cdots, 2 N-1,
$$

for $n$ even and

$$
\frac{\sinh m \sigma}{m \sigma}=\frac{1}{2} \beta_{0}+\sum_{i=1}^{N} \beta_{i} \cosh m \sigma x_{i}, \quad m=0,1, \cdots, 2 N,
$$

for $n$ odd.

We can now proceed to develop the asymptotic solution to these equations as $\sigma \rightarrow \infty$. The main result is given in the following theorem.

THEOREM 3. Let $x_{1}=\min _{i}\left(x_{i}\right)$. Then as $\sigma \rightarrow \infty$ we have for $n=2 N$

$$
x_{1}=1-\frac{\ln \left(N^{2} \sigma\right)}{\sigma}+O\left(\sigma^{-2}\right) \text {, }
$$


and for $n=2 N+1$

$$
x_{1}=1-K / \sigma+O\left(e^{-\sigma}\right)
$$

where $K>0$ is a constant depending only on $N$.

Consider first the case $n$ even. As $\sigma \rightarrow \infty$ we have $\sinh \sigma \eta \sim \cosh \sigma \eta \sim \frac{1}{2} e^{e \text { for }}$ any $\eta>0$. Thus, the dominant solution to (10) is obtained from

$$
1=\sum_{i=1}^{N} \beta_{i}, \quad \frac{1}{m \sigma}=\sum_{i=1}^{N} \beta_{i} \phi_{i}^{m}, \quad m=1,2, \cdots, 2 N-1,
$$

where $\phi_{i}=\exp \left(\sigma\left(x_{i}-1\right)\right)$. It should be noted this is precisely the system of equations which results from the method of undetermined coefficients applied to (9) with $f \in\left\{1, e^{\sigma x}, \cdots, e^{(2 N-1) \sigma x}\right\}$. It then follows from [1] that the $\phi_{i} \in(0,1)$ and are distinct. We can reduce the problem of solving this system to finding the roots $\phi_{i}$ of a polynomial using Prony's method of exponential interpolation [6, p. 378]. The $\beta_{i}$ are then obtained by solving a system of linear equations with a Vandermonde coefficient matrix (which is nonsingular).

Suppose the polynomial is $\alpha_{N} \phi^{N}+\cdots+\alpha_{1} \phi+1$. Then the coefficients must satisfy the system of linear equations

$$
\sum_{i=1}^{N} H_{i j} \alpha_{i}=-p_{i}, \quad i=1, \cdots, N,
$$

where $H_{i j}=1 /(i+j-1)$ are the elements of an $N$ th order Hilbert matrix, $p_{1}=\sigma$, and $p_{i}=1 /(i-1)$ for $i \geqq 2$. The inverse of the Hilbert matrix is known to have elements [7]

$$
H_{i j}^{-1}=(-1)^{i+i} \frac{(N+i-1) !(N+j-1) !}{(i+j-1)[(i-1) !(j-1) !]^{2}(N-i) !(N-j) !} .
$$

Note that all the $\alpha_{i}=O(\sigma)$ as $\sigma \rightarrow \infty$. From this it is evident that there are no roots of the polynomial going to zero faster than $1 / \sigma$. Suppose there is a root $\phi_{1}=C / \sigma+$ $O\left(1 / \sigma^{2}\right)$. Then since $\alpha_{1}=-N^{2} \sigma+O(1)$ the polynomial equation is $1-N^{2} C+$ $O(1 / \sigma)=0$, whence $C=1 / N^{2}$. There is no other root of order $1 / \sigma$; as a matter of fact, the remaining $\phi_{i}=O(1)$. Expressing $x_{1}$ in terms of $\phi_{1}$ then gives the result stated in the theorem.

In the case of $n$ odd the dominant system of equations derived from (11) is

$$
\beta_{0}=2\left(1-\sum_{i=1}^{N} \beta_{i}\right), \quad \frac{1}{m \sigma}=\sum_{i=1}^{N} \beta_{i} \phi_{i}^{m}, \quad m=1,2, \cdots, 2 N .
$$

In this case the system of equations involving the $\phi_{i}$ result by applying the method of undetermined coefficients to (9) with $f \in\left\{e^{\sigma x}, \cdots, e^{2 N \sigma x}\right\}$, and so we know from [1] that the $\phi_{j}$ are distinct and lie in the interval $(0,1)$. We again use Prony's method to derive a polynomial with roots $\phi_{i}$, but in this case it is convenient to assume that the polynomial is $\phi^{N}+\tilde{\alpha}_{N} \phi^{N-1}+\cdots+\tilde{\alpha}_{2} \phi+\tilde{\alpha}_{1}$. The coefficients then satisfy

$$
\sum_{i=1}^{N} H_{i j} \tilde{\alpha}_{i}=-\frac{1}{N+i}, \quad i=1, \cdots, N .
$$

Note that the coefficients depend only on $N$. Let $\phi_{1}$ be the smallest root of the polynomial and define $K=\ln \left(1 / \phi_{1}\right)>0$. The result stated in the theorem follows: 
It is interesting to note that the Christoffel numbers $\beta_{i}=O(1 / \sigma)$ for $j \geqq 1$ and $\beta_{0}=2-O(1 / \sigma)$ with $n$ odd, whereas the $\beta_{i}=O(1)$ for $n$ even.

We see from this theorem that the nonzero nodes of the quadrature formula (1) tend to coalesce at the endpoints of the interval as $\sigma \rightarrow \infty$. Thus, for any sufficiently large $\sigma$ the nodes in (1) must lie outside the Legendre nodes. This leads us to

Conjecture 2. Each nonzero node $x_{i}$ of the quadrature formula (1) moves monotonically outward from the Legendre node toward the endpoints of the interval $[-1,1]$ as $\sigma$ increases from zero toward infinity. The movement is not strictly monotone at $\sigma=0$ due to Theorem 2 .

This conjecture has also been tested with numerical experiments.

Having discussed in some detail the qualitative nature of the quadrature formula, let us now turn to the actual construction of the nodes and weights. We will treat $\omega$ as real in the development, though the results are valid also for imaginary $\omega$. The scheme we propose is based on the three-term recursion relation

$$
p_{r+1}=y p_{r}-C_{r} p_{r-1},
$$

with $p_{0}=1, p_{1}=y$, and $C_{r}=\left(p_{r}, p_{r}\right) /\left(p_{r-1}, p_{r-1}\right)$, which follows from the theory of orthogonal polynomials.

Consideration of the recursion relation shows that the polynomials are of the form

$$
\begin{gathered}
p_{2 i}=y^{2 i}+A_{i}^{(2 i)} y^{2 i-2}+\cdots+A_{2}^{(2 i)} y^{2}+A_{1}^{(2 j)} \\
\left.p_{2 i+1}=y\left[y^{2 i}+B_{i}^{(2 i+1)}\right) y^{2 i-2}+\cdots+B_{2}^{(2 i+1)} y^{2}+B_{1}^{(2 j+1)}\right] .
\end{gathered}
$$

We can now substitute polynomials of this form into the recursion relation and collect like powers of $y$ to show that

$$
\begin{aligned}
A_{i}^{(2 i)} & =B_{i-1}^{(2 j-1)}-C_{2 i-1} A_{i}^{(2 j-2)}, \quad i=1,2, \cdots, j, \\
B_{i}^{(2 j+1)} & =A_{i}^{(2 i)}-C_{2 i} B_{i}^{(2 j-1)},
\end{aligned}
$$

where we define $B_{0}^{(2 m+1)}=0$ for all $m \geqq 0$. Since $\left(p_{r}, p_{r}\right)=\left(y^{r}, p_{r}\right)$ we can also evaluate the $C_{r}$ in terms of the polynomial coefficients once we know the inner products $(1,1), \cdots,\left(y^{n}, y^{n}\right)$. In this way finding the coefficients of $p_{n}(y)$ is reduced to a numerical problem.

The inner products can also be evaluated recursively. If we let $F=G=y^{m}$, $m=0,1, \cdots, n$, in (7) and make the transformation $y=s^{-1} \sin \phi$ we find

$$
\left(y^{m}, y^{m}\right)=s^{-2 m-1} \int_{-\omega / 2}^{\omega / 2} \sin ^{2 m} \phi d \phi=\frac{1}{2 m s^{2}}\left[(2 m-1)\left(y^{m-1}, y^{m-1}\right)-2 \cos \frac{\omega}{2}\right] \text {, }
$$

after an integration by parts. For large $\omega$ it is appropriate to begin the recursion with $(1,1)=\omega / s$, but for small $\omega$ a forward recursion suffers large round-off errors. We can avoid the difficulty by using a backward recursion for small $\omega$ with the initial value $\left(y^{n}, y^{n}\right)$ obtained by an alternate procedure. For example, we can use the backward recursion relation to show that

$$
\left(y^{n}, y^{n}\right)=\frac{2 \cos (\omega / 2)}{2 n+1}\left\{1+\frac{2 n+2}{2 n+3} s^{2}+\frac{2 n+2}{2 n+3} \frac{2 n+4}{2 n+5} s^{4}+\cdots\right\}
$$

for $|s|<1$. Note that the series is the hypergeometric function $F\left(n+1,1 ; n+3 / 2 ; s^{2}\right)$. 
The inner product $\left(y^{n}, y^{n}\right)$ can also be evaluated by Gauss-Legendre quadrature for small $s$, since the integrand is well approximated by a polynomial.

After the coefficients of $p_{n}(y)$ have been found, the polynomial can be factored to determine its zeros $y_{i}$. The nodes and Christoffel numbers are then

$$
x_{i}=\frac{2}{\omega} \sin ^{-1}\left(s y_{i}\right), \quad \beta_{i}=\frac{2 s}{\omega} \frac{\left(p_{n-1}, p_{n-1}\right)}{p_{n}^{\prime}\left(y_{i}\right) \cdot p_{n-1}\left(y_{i}\right)} .
$$

In summary, we believe that the parametrized quadrature formula we have been discussing provides a valuable unifying concept as mentioned in the introduction. Furthermore, its flexibility should make it possible on occasion to obtain an optimal matching of formula to problem.

Boeing Company

Renton, Washington 98055

1. A. C. R. Newbery, "Some extensions of Legendre quadrature," Math. Comp., v. 23, 1969 , pp. 173-176.

2. E. IsAacson \& H. B. Keller, Analysis of Numerical Methods, Wiley, New York, 1966. MR 34 \#924.

3. C. Lanczos, Applied Analysis, Prentice-Hall, Englewood Cliffs, N. J., 1956. MR 18, 823.

4. W. GAUTSCHI, "Numerical integration of ordinary differential equations based on trigonometric polynomials," Numer. Math., v. 3, 1961, pp. 381-397. MR 25 \#1647.

5. G. Birkhoff, D. M. Young \& E. H. Zarantonello, Numerical Methods in Conformal Mapping, Proc. Sympos. Appl. Math., vol. 4, Fluid Dynamics, McGraw-Hill, New York, 1953, pp. 117-140. MR 15, 258.

6. F. B. HILDEBRAND, Introduction to Numerical Analysis, McGraw-Hill, New York, 1956. MR 17, 788.

7. M. MARcus, "Basic theorems in matrix theory," Nat. Bur. Standards Appl. Math. Ser., v. 57, 1960. MR $22 \# 709$. 